\title{
Multimodal Image Fusion based on Hybrid of Hilbert Transform and Intensity Hue Saturation using Fuzzy System
}

\author{
Munish Rehal \\ Scientist/Engineer 'SE' \\ Semi-Conductor Laboratory, Mohali
}

\author{
Akhil Goyal \\ Centre for Development of Advanced Computing, \\ Mohali
}

\begin{abstract}
A fused image was produced from the Standard Image Fusion in that in every source image from a set of pixels in which every pixel is established. Several mechanisms of image fusion have been detected which is useful to fuse the images and the resultant image is much meaningful. In existing work, this has been seen that in the case of Position Emission Tomography (PET) images the traditional technique is not able to give the informative output due to the presence of a non-informative part of the information in the data. So, when these images are further fused with MRI images then it will affect the final output. Another limitation is the lack of optimization approach as existing traditional approach have low solving precision, bad local searching ability, and slow convergence. This creates a need of proposing a new approach in which a combination of 2-d Hilbert transform and Intensity Hue Saturation method is used for fusing the images. In traditional pixel-level mechanisms an addition, subtraction, multiplication, and weighted average are used that are easy and minimally accurate. The Intensity Hue Saturation method is also popular for image fusions due to its ability to give a high resolution of images. Then, the region of interest extraction approach is applied to PET images that remove its drawback of having irrelevant information. Further, the Gray wolf optimization approach Is applied to the outcome for more accurate results.
\end{abstract}

\section{Keywords}

Medical image fusion, PET image, MRI image, Gray wolf optimization, Intensity Hue Saturation, 2-D Hilbert transform

\section{INTRODUCTION}

Image fusion is an approach of combining the important and complementary information of several images without affected the information in it [1-2]. In this, several images as input of same or different domains are merged to make a new single image that contains relevant, consistent, reliable, and larger information as compared to individual input images [3]. The image achieved from it will be more complete, descriptive, accurate, and informative than original images on the source side [4]. The motive behind performing image fusion is to integrate the low detail multisensory images taken from common sense for obtaining a hybrid or fused image that has high spatial resolution [5]. It is one of the most effective approaches in the extraction of informative values from a large number of images that optimally improve spatial and spectral resolution. Image fusion also helps in reducing the amount of information by representing it in a useful and more descriptive way. Further, it converts the multimodal images' useful information into a single one. By bringing improvement in different domains of imaging approaches and modern instrumentation the multisensory system becomes useful in various applications such as military applications, automatic vehicular systems, medical imaging, machine vision, remote sensing, and high-speed object tracking. In real-time applications such as advancement by image, fusion makes it a more powerful and efficient tool for increasing the perception of machine and human along with the reduction in requirement of storage space and cost minimization [6]. Medical image fusion is an application of image fusion that is developing and emerging rapidly for handling the different issues retrieved from multimodal medical images. While fusion medical images, several images are taken from either single or multiple imaging tools then combined its information significantly into a single image that helps in describing the scene more efficiently by recollecting the useful information from it. It helps in improving image reliability and reducing the redundancy and cost of storage. Along with it, the quality gets increased which gives a clear visual effect of medical images for medical problems analysis, diagnosis, and treatment [7]. This approach has gained importance in the field of tumor diagnosis in the breast, mouth, and brain. In the medical image fusion process, multiple images are acquired using either a common source or different modalities of imaging. Multimodal modal image fusion is defined as the fusion of medical images using two or more tools of imaging. Also, it is a concept of improving the content of images and their quality by merging all the captured images using various imaging tools. Some of these imaging tools are PET, X-ray, Magnetic Resonance Imaging (MRI), and Computed Tomography. All these tools give various spectral features of images and combined them. As the use of a single image not able to fulfills the requirement of physicians in case of clinical diagnosis. So, to get accuracy in diagnosis there is a need of fusing the features of more than one image. This helps in removing the diagnosis limitation of a single sensor image.

\section{MOTIVATION OF MEDICAL IMAGE FUSION}

With continuous development in instrumentation and image technology, image fusion has come as a powerful tool for the enhancement of image features and improving the quality that makes the outcomes more descriptive and clear. In an automatic vehicular system, two or more two images related to complementary information are combined for accurate detection. In medical assessment, a correct diagnosis can be done by combining the specific quality of each image. Using this image fusion one can also detect a high-speed object, hazardous weapon, and obstacle. It also plays an important role in tracking the path of the vehicle in rain or fog, detection of airline track in hostile climatic condition and automatic robotics and machines. The main aim of using image fusion in medical are: 
- Better diagnosis of diseases

- Reduction of storage space

- Enable distant effective and accurate assessment

- $\quad$ Making more effective clinical instruments

- Enhance the information content of a single image

\section{EXISTING WORK}

In medical treatment and diagnostics, an important role is played by multimodal medical image fusion. There is the problem of high complexity and spatial inconsistency in CT, DWT, NCST, and CVT image fusion methods based on the transform domain. There is a problem of halo artifacts and contrast reduction in spatial domain image fusion technique based on a guided filter. So, this is modified by Pritika, et al. (2015), for which they have used Gaussian decomposition along with local average energy and saliency maps based on the average gradient for detail and base layer [8]. The experiment results performed on it shows the effectiveness in preserving contrast and fine details as compared to the existing fusion scheme. K. P. Indira, et al. (2015), has worked on fusion methods for eight sets of Discrete Wavelet Transform (DWT) based PET and CT images [9]. The PET images are used for checking the function of the brain with low spatial resolution and CT image is sensitive to bones. The DWT is considered the most popular tool for image processing. For fusion in low-frequency coefficients, a maximum and average rule are applied and contrasts, maximum and gradient rule are applied for high-frequency coefficients. For analysis purpose, various fusion method results are obtained that shows average fusion rule is the best method for low frequency coefficients and gradient fusion rule is the best for high frequency coefficients. Other thing observed is the reduction in standard deviation and root mean square error values for average-gradient method and Peak signal to noise ratio and entropy values get increased for the same.

Xingbin Liu, et al., (2015), have proposed a fusing undersampled $\mathrm{k}$-space data compressing sensing based novel multi-modality medical image fusion method [10]. For kspace data undersampled low and high frequency subbands a combined fusion strategy was applied for transferring the structural information from the source to fused images. Further, from fused subband data a fused imaged is reconstructed with conjugate gradient method. The simulation performed on it shows that there is substantial reduction in sampling data and clinical diagnosis demand is fulfilled using it. Qiang Cao, et al., (2017), have presented a new GPU accelerated non-subsampled shearlet transform and 2D PCA based image fusion method [11]. In this work, the subbands coefficients are divided into block after the transform to keep the pixel distribution smooth in fused image. In order to reduce the distortion in changes in existing coefficients a regional and global fusion strategy was used. The simulation results obtained by experiment shows the reduction the distortion in coefficients changes along with improvement on the quality of fused image as compared to other existing multiscale transform based methods.

Behzad Rezaeifar, et al., (2017), have proposed a novel approach to multimodal medical image fusion [12]. As, in everyday clinical practices medical imaging has become a common part. Due to this, a great progress has been done in this field but still not a single modality able to represent all aspects of human body. For instance high resolution for soft tissue is provided by MRI and dense structures are viewed using CT. In the proposed work, firstly the sources images are decomposed using surfacelet transform then their high and low frequency coefficients are combined effectively [13]. In the end, the fused image was obtained using inverse transform. To test the applicability, the proposed method was tested and compared to counterpart algorithms that show an improved quality of results using proposed one. Harmeet Kaur, et al., (2018), have worked on multi-modality mages fusion to get accurate and efficient low cost high end treatment [14]. The medical images are considered as main source of information for treatment and diagnostic planning. In their work, they have used Harvard database and the modality of medical images is different depending of their modalities through which it comes that can be MRI, PET and $\mathrm{CT}$. The fusion of these different modalities is done to get best information in fused image and to get it a forward fuzzy logic interface system was used by them for image fusion. The input images considered in proposed work was MRI and CT that are fused using fuzzy logic. It is tested in terms of MSE, SNR and PSNR that gives improves information using proposed work as compared to wavelet based fusion method.

Ebenezer Daniel, (2018), have proposed a Homomorphic wavelet fusion known as Homomorphic Wavelet Fusion or OHWF using Genetic - Grey Wolf Optimization (HGGWO) Algorithm [15]. The OHWF is the combination of wavelet domain and logarithmic information of input images and input image multi-level decomposition features comes under wavelet based homographic fusion. In the proposed work, they have given the approximation coefficients of modality 1 and optimum scaled detailed coefficients of modality 2 to adder 1 . And, the approximation coefficients of modality 2 and optimum scaled detailed coefficients of modality 1 are added together in adder 2 . Then the resultants of these two adders are fused using averaging rule based on pixel. In the end they have tested their proposed approach for MR-CT, MR T1-T2, MR-SPECT and MR-PET image fusion in terms of different evaluation parameters. Further, the modification in grey wolf optimization was done by applying genetic operator and results shows that the proposed approach perform well in terms of functional and structural information in the fused image. Ming Yin, et al., (2019), have proposed a method in which to get multi-direction and multiscale representation NSST decomposition was performed on source images [16]. The parameter-adaptive pulse-coupled neural network (PAPCNN) model was used for fusing the high-frequency bands in which the input band was used for estimating the additivity of PCNN parameters. Through novel strategy a low frequency bands wre merged that addresses the detail extraction and energy preservation two critical issues of medical image fusion. In the end inverse NSST was applied on low and high frequency bands for reconstruction of fused image. The MRT1 and MR-T2, positron emission tomography and MR, MRI and CT , and single photon emission CT and MR four medical image fusion problems was considered by them for checking the effectiveness of the proposed method. The results obtained on it shows that the method is effective in both objective assessment and visual quality as compared to existing nine medical image fusion methods. Xuecan Liang, et al., (2019), have proposed multi-layer concatenation fusion network (MCFNet) that is an end to end deep learning network for reconstruction of images, feature fusion and extraction without designing rules of fusion and feature extraction [17]. The proposed network connects the feature map of every layer 
present in reconstruction network of image with source map of having same resolution due to down sampling. During the supplement process of two down-sampling the spatial information get lots in the fuse medical images and further the fast up-convolution was used at image reconstruction stage of up- sampling operation that reduces the checkerboard effects of traditional deconvolution [18]. For testing purpose different clinical medical images was considered and fused using traditional methods of image fusion that shows an achievement of improving fusion results in terms of objective evaluation and visual quality. B. Ashwanth, et al., (2020), have used Stationary Wavelet Transform (SWT) and Discrete wavelet transform (DWT) for proposing a PET and MRI medical image fusion in the transform domain [19]. After that performed energy and edge rule of decomposed bands of above-mentioned image fusion approaches. The entropy is used for assessing the performance that shows a better-fused image can be obtained using a new rule of fusion [21].

\section{PROPOSED WORK AND OBJECTIVES}

After reviewing various works done by different researchers and coming across with issues existing, I have concluded that there is a need of introducing mechanisms that able to overcome the limitations of traditional work. So, to achieve this new mechanism of ROI is applied on PET images for extracting the region of interest from these images. The idea behind performing this task was to get an only informative part for the fusion process. After applying ROI to PET images an improved fusion method is applied to the MRI and PET image information obtained after passing through ROI and get the final fused image. The objectives of the proposed work are:

1. To study the various approaches used for image fusion.

2. To implement the region of interest approach to PET images for avoiding the unnecessary information

3. To apply the final stage coefficients fusion based on the Fuzzy rule approach.

4. To conduct a comparative analysis of the proposed fusion approach with traditional HT-HIS and other models

\section{RESULTS AND DISCUSSION}

The proposed work implements the image fusion to the medical images and this section of the study shows the results that are acquired after simulating the proposed image fusion technique in MATLAB. Figure 1 and 2 shows two input images namely MRI and PET image that has been considered for proposed work analysis.

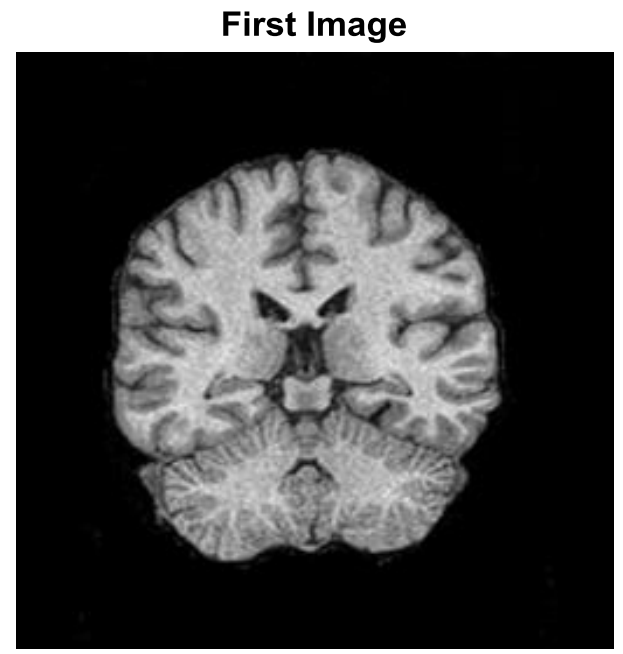

Fig 1: Input MRI image

The above-shown Figure 1 and Figure 2 show the first input MRI image and the PET image of the first input MRI image. Here the MATLAB 2015b is utilized for simulation analysis.

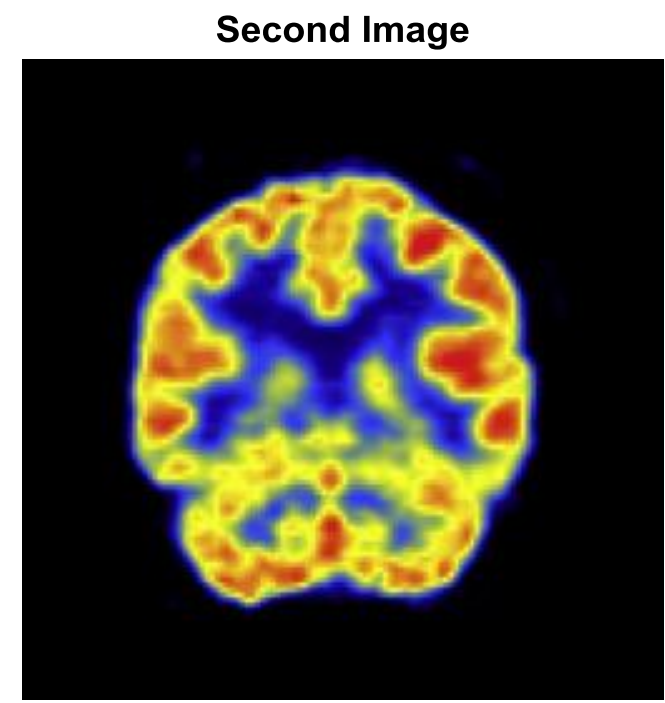

Fig 2: Input PET image

On this PET image, we have applied RGB to IHS then perform ROI on it and apply the IHS approach and on the I component I have applied 2-DHT and taken PET coefficients out from it. The final image obtained from it is shown in below figure 3 .

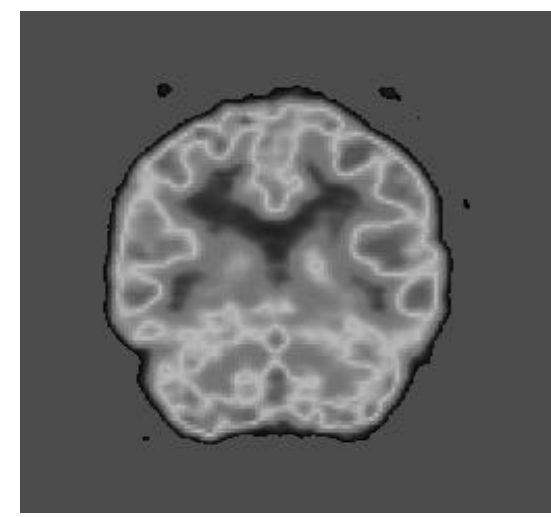

Fig. 3: PET image after processing 
However, graph shown in figure 4 demonstrate the fusion of both MRI and PET images. The first input taken is MRI image and second one is PET image of the first MRI image. On input image, we have applied 2-DHT and taken their MRI coefficients and done fusion using fuzzy approach

\section{Fused Image}

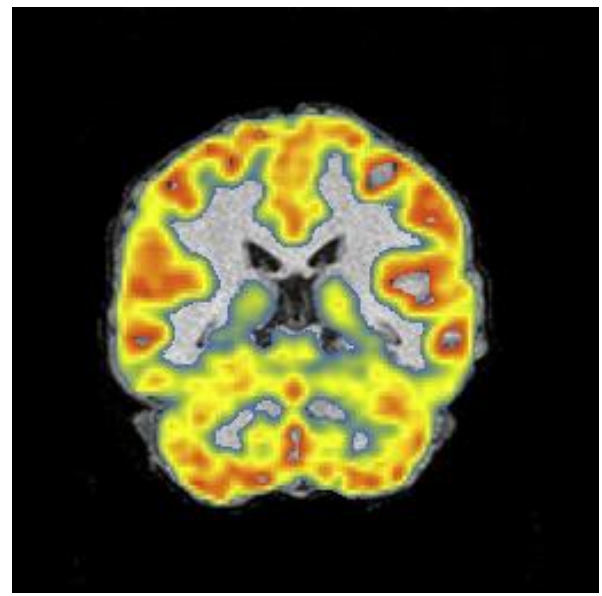

Fig. 4: Fused image

\section{Comparative Results:}

Figure 5 shows the graph of comparative analysis of results obtained using proposed and traditional image fusion techniques. The comparison analysis is done in the terms of discrepancy. Discrepancy refers to the evaluation of the capability of a fused image to preserve the spectral features of the input image. It can be measured as follows:

$$
D_{k}=\frac{1}{M * N} \sum_{i=1}^{M} \sum_{j=1}^{N}\left|f_{k}(x, y)-f_{2 k}(x, y)\right|
$$

Where, $f_{k}(x, y)$ and $f_{2 k}(x, y)$ refers to the pixel values for fused images concerning the $\mathrm{x}$ and $\mathrm{y}$ pixel. The $\mathrm{y}$ axis in the graph refers to the values for the discrepancy that ranges from 0 to 25 . The values for the discrepancy are shown in table 1 .

This is the comparison graph of various approaches in terms of discrepancy. The value of discrepancy should be very low and in our case, it is 9.09 .

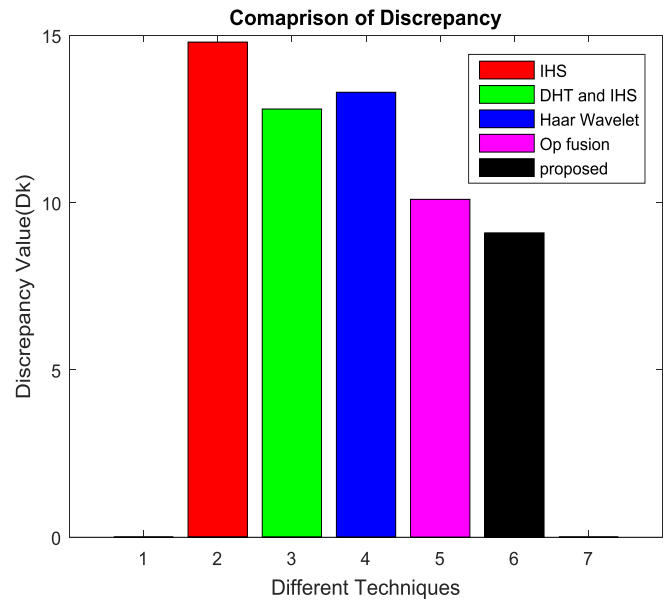

Fig. 5: Comparison of Discrepancy
Table 1. Values of Discrepancy Value for various image fusion techniques

\begin{tabular}{|l|l|}
\hline Techniques & Discrepancy Value \\
\hline HIS & 14.8 \\
\hline DHT and HIS & 12.8 \\
\hline Gradient pyramid & 15.9 \\
\hline FSD Pyramid & 16.1 \\
\hline 2DHT & 20.3 \\
\hline Haar Wavelet & 13.3 \\
\hline Proposed Work & 9.09 \\
\hline
\end{tabular}

The graph in figure 6 represents the comparison of IHS, DHT-IHS, Gradient Pyramid, FSD Pyramid, 2DHT, Haar Wavelet and Proposed work on the basis of the average gradient. Average Gradient is a performance metrics that defines the capability of the fused image to preserve the spatial features of the original image.

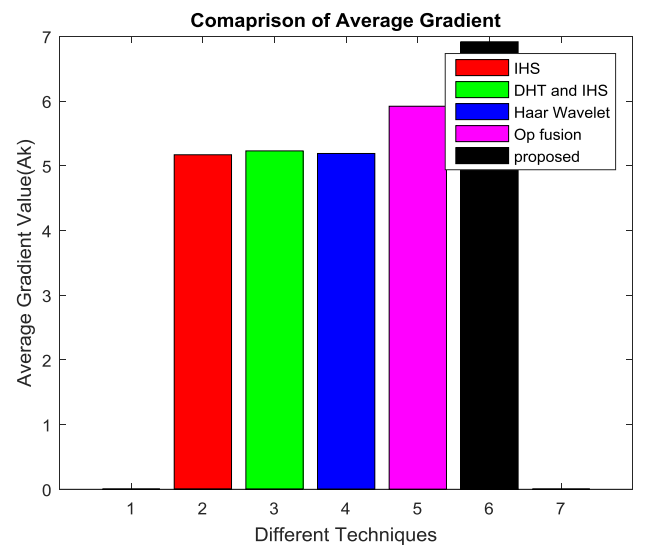

Fig. 6: Comparison of Average Gradient

It is evaluated by using the following formulation:

$$
\begin{aligned}
A G_{K}=\frac{1}{(M-1)} & *(N-1) \\
& * \sum_{i=1}^{M} \sum_{j=1}^{N} \sqrt{\frac{\left(\frac{\partial f}{\partial x}\right)^{2}+\left(\frac{\partial f}{\partial y}\right)^{2}}{2}}
\end{aligned}
$$

This is the comparison graph of various approaches in terms of the average gradient. The value of the average gradient should be very high and in our case, it is 6.91. The graphs depict that the proposed work has a higher average gradient in comparison to the traditional image fusion techniques. The facts and observations are shown in table 2 . 
Table 2. Values of Average Gradient for various image fusion techniques

\begin{tabular}{|c|c|}
\hline \\
\hline Techniques & Average Gradient \\
\hline HIS & 5.17 \\
\hline DHT and HIS & 5.23 \\
\hline Gradient pyramid & 4.66 \\
\hline FSD Pyramid & 4.73 \\
\hline $2 \mathrm{DHT}$ & 4.89 \\
\hline Haar Wavelet & 5.19 \\
\hline Proposed Work & 6.91 \\
\hline
\end{tabular}

The analyses of the overall performance of the proposed and traditional techniques are shown in the graph of figure 7 . The overall performance is evaluated based on the average gradient and discrepancy value of the techniques. The following mathematical equation is followed to evaluate the overall performance of the techniques.

$$
O . P=\frac{\sum_{K}\left|D_{K}-A G_{K}\right|}{3}
$$

Where, $\mathrm{k}$ can be red, green, or blue.

This is the comparison graph of various approaches in terms of overall performance. The value of overall performance should be very low and in our case, it is 0.86 .

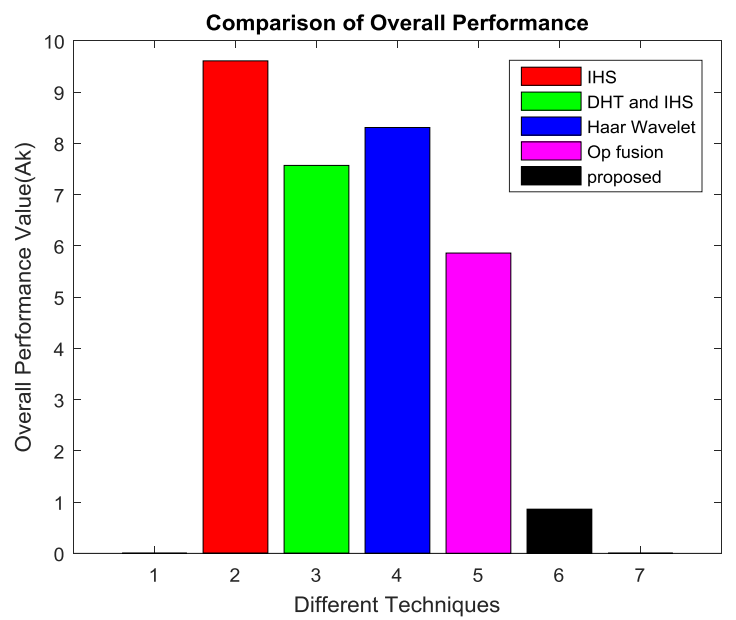

Fig. 7: Comparison of Overall Performance

Table 3. Comparative table of results

\begin{tabular}{|l|l|l|l|}
\hline Approach & Discrepancy & $\begin{array}{l}\text { Average } \\
\text { Gradient }\end{array}$ & $\begin{array}{l}\text { Overall } \\
\text { perfor } \\
\text { mance }\end{array}$ \\
\hline HIS & 14.8 & 5.17 & 9.61 \\
\hline $\begin{array}{l}\text { DHT } \\
\text { AND HIS }\end{array}$ & 12.8 & 5.23 & 7.57 \\
\hline $\begin{array}{l}\text { Haar } \\
\text { wavelet }\end{array}$ & 13.3 & 5.19 & 8.31 \\
\hline Op fusion & 10.1 & 5.92 & 5.86 \\
\hline $\begin{array}{l}\text { Proposed } \\
\text { work }\end{array}$ & 9.09 & 6.91 & 0.86 \\
\hline
\end{tabular}

\section{CONCLUSION}

Medical images have been used for making decisions by detecting diseases in the patient using various medical images that can CT scan, X-Ray, MRI, PET, and other types of images. As it is concerned with the life of someone so the decision driven should be much accurate, efficient and less time-consuming. For this purpose, several image processing approaches have been introduced for enhancing the quality of medical images without destroying the original content of the image. From all images, fusion is considered as a process of combining two not-so-good images and form an individual enhanced image from it. In existing work, various waveletbased approaches have been introduced but it is not able to give good results due to low quality of images and high noise present in it. Considering these factors a new approach has been proposed in which initially Hybrid fast Fourier transform is used to extract features from a set of images along with intensity Hue saturation approach then acquired the optimum solution by applying Gray wolf optimization algorithm on it. The proposed method has been tested on MATLAB software that shows improved results using the proposed approach as compared to the traditional one that has been used in existing works. The parameters that have been used for analysis purpose are Entropy, Standard deviation, Mutual information and $\mathrm{Q}(\mathrm{AB} / \mathrm{F})$ that shows an improvement from existing techniques used for same work.

\section{REFERENCES}

[1] Shutao Li, "Multifocus image fusion using artificial neural networks", Pattern recognition Letters, Vol. 23, No. 8, pp. 985-997, June 2002

[2] R. Maruthi, "Multi Focus Image Fusion Based On The Information Level In The Regions Of The Images", Journal of Theoretical and Applied Information Technology, 2007, pp. 80-85.

[3] Jun Kong, "Multi-focus Image Fusion Using Spatial Frequency and Genetic Algorithm", IJCSNS International Journal of Computer Science and Network Security, Vol. 8, No.2, pp. 220-224, February 2008

[4] Anjali Malviya and S. G. Bhirud, "Image Fusion of Digital Images", International Journal of Recent Trends in Engineering, Vol. 2, No. 3, pp. 146-148, November 2009

[5] Qiang Fu, "Multi-focus Image Fusion Algorithms Research Based on Curvelet Transform", Genetic and Evolutionary Computing, 2009. WGEC '09. 3rd International Conference on, pp. $442-446$, February 2010

[6] Wu Yiqi, "Multilevel and Multifocus Image Fusion Based on Multi-Wavelet Transformation" Computer Network and Multimedia Technology,. CNMT 2009. International Symposium on, January 2010, pp. 1-4.

[7] A.Uma Maheshwari, "Image fusion techniques", International Journal of Recent Research and Applied Studies, Vol. 4, No. 1, pp. 69-74, 2010

[8] Pritika, Sumit Budhiraja, "Multimodal medical image fusion using modified fusion rules and guided filter", International Conference on Computing, Communication and Automation (ICCCA), 2015, pp. 1067-1072.

[9] K.P.Indira, Dr.R.Rani Hemamalini, "Impact of CoEfficient Selection Rules on the Performance of DWT Based Fusion on Medical Images", International Conference on Robotics, Automation, Control and Embedded Systems - RACE, 2015, pp. 1-8. 
[10] Xingbin Liu, Huiqian Du, Jiadi Bei, Wenbo Mei, "Compressed Sensing Based Multi-modal Medical Image Fusion Using a Combined Fusion Strategy", 8th International Conference on BioMedical Engineering and Informatics (BMEI), 2015, pp. 85-89.

[11] Qiang Cao, Baosheng Li, Liyuan Fan, "Medical Image Fusion Based on GPU Accelerated Nonsubsampled Shearlet Transform and 2D Principal Component Analysis", IEEE 2nd International Conference on Signal and Image Processing, 2017, pp. 203-207.

[12] Behzad Rezaeifar, Mahdi Saadatmand-Tarzjan, "A New Algorithm for Multimodal Medical Image Fusion Based on the Surfacelet Transform", 7th International Conference on Computer and Knowledge Engineering (ICCKE), 2017, pp. 396-400.

[13] Y. Kumar, K. Kaur and G. Singh, "Machine Learning Aspects and its Applications Towards Different Research Areas," 2020 International Conference on Computation, Automation and Knowledge Management (ICCAKM), 2020, pp. $150-156$

[14] Harmeet Kaur, Satish Kumar, "Fusion of Multi-Modality Medical Images: A Fuzzy Approach", IEEE 3rd International Conference on Computing, Communication and Security (ICCCS), 2018, pp. 112-115.

[15] Ebenezer Daniel, "Optimum Wavelet Based Homomorphic Medical Image Fusion Using Hybrid Genetic - Grey Wolf Optimization Algorithm", IEEE Sensors Journal, 2018, pp. $6804-6811$.

[16] Ming Yin, Xiaoning Liu, Yu Liu, Xun Chen, "Medical Image Fusion With Parameter-Adaptive Pulse CoupledNeural Network in Nonsubsampled Shearlet Transform Domain", IEEE Transactions On Instrumentation And Measurement, 2019, pp. 49-64.

[17] Xuecan Liang, Pengyu Hu, Liguo Zhang, Jianguo Sun, Guisheng Yin, "MCFNet: Multi-Layer Concatenation Fusion Network for Medical Images Fusion", IEEE Sensors Journal, 2019, pp. 7107-7119.
[18] K. Kaur and Y. Kumar, "Swarm Intelligence and its applications towards Various Computing: A Systematic Review," 2020 International Conference on Intelligent Engineering and Management (ICIEM), 2020, pp. 5762

[19] B. Ashwanth, K.Veera Swamy, "Medical Image Fusion using Transform Techniques", 5th International Conference on Devices, Circuits and Systems (ICDCS), 2020, pp. 303-306.

[20] Jigneshkumar M.Patel, Mehul C.Parikh, "Medical Image Fusion Based On Multi-Scaling (DRT) and MultiResolution (DWT) Technique", International Conference on Communication and Signal Processing, 2016, pp. 654-657.

[21] Ebenezer Daniel, "Optimum Wavelet Based Homomorphic Medical Image Fusion Using Hybrid Genetic - Grey Wolf Optimization Algorithm", IEEE Sensors Journal, 2018, pp. $6804-6811$.

[22] Yong Yang, Yue Que, Shuying Huang, Pan Lin, "Multimodal Sensor Medical Image Fusion Based on Type-2 Fuzzy Logic in NSCT Domain”, IEEE Sensors Journal, 2016, pp. 3735 - 3745 .

[23] Kaul S., Kumar Y. "Nature-Inspired Metaheuristic Algorithms for Constraint Handling: Challenges, Issues, and Research Perspective", In: Kulkarni A.J., Mezura-Montes E., Wang Y., Gandomi A.H. Krishnasamy G. (eds) Constraint Handling in Metaheuristics and Applications, Springer, 2020

[24] Sunil Kumar Panjeta, "A Survey on Image fusion Techniques used to Improve Image Quality" International Journal of Applied Engineering Research, Vol.7, No.11, 2012

[25] C. Morris and R.S. Rajesh, "Two stage spatial domain image fusion techniques", ICTACT journal on image and video processing, Vol. 5, No. 1, Pp. 8895-898, August 2014 